\title{
Study on the Physical Model and Experiments of MIMO Theory
}

\author{
Haiyang $\mathrm{Fu}$ \\ College of Telecommunication and Information \\ Engineering, Nanjing University of Posts and \\ Telecommunications \\ Nanjing, P. R. China \\ e-mail: fuhy@njupt.edu.cn
}

\author{
Bin Hu, Xiangdong Jia, Hui Fang \\ College of Telecommunication and Information \\ Engineering, Nanjing University of Posts and \\ Telecommunications \\ Nanjing, P. R. China \\ e-mail: hubin0708274@163.com
}

\begin{abstract}
The present researches of the MIMO theory only proved that channel capacity (CC) could be increased on the setting mathematical model in base-band by the space division multiplex (SDM) principle. But the reasonable physical model matching to that mathematical model has not been given by those researches, resulting in that the SDM could not be realized in MIMO system. So that the CC formula of MIMO system deduced by that mathematical model could not be realized physically. The infeasibility of MIMO system would be discussed in the paper in the aspects of physical characteristic of antenna, radio beam patterns of many kinds of antenna array, the un-reasonability of the MIMO physical model, the experiments of MIMO, and etc.
\end{abstract}

Keywords-MIMO; Shannon Formula; Channel Capacity; Physical Characteristic of Antenna

\section{INTRODUCTION}

The requirements for the channel capacity (CC) or bit rate $R_{b}$ of communication are increased quickly by the modern wireless communication. According to the Shannon formula which gives the ultimate value of CC, it is known that the CC is decided by the channel bandwidth and the receiving signal to noise ratio (SNR), which are the only two physical parameters. So it is the main goals of the new technology of modern wireless communication that how to make use of more narrow channel bandwidth, or make one's best to obtain larger receiving SNR under the condition of maintaining the transmitting power unchanged to obtain larger CC. Generally speaking, it could be thought as two basic issues of how to improve the spectrum efficiency $\eta_{f}$ and power efficiency $\eta_{p}$ [1]. However, the criteria of $\eta_{p}$ scarcely be considered in the present communication theory, which should be corrected.

The first definition of $\eta_{p}$ increasing is the decreasing of the needed signal power of receiving or SNR for the transmission of the same $R_{b}$, the second definition of $\eta_{p}$ increasing given by us is that to gain better receiving SNR under the condition of transmitting power unchanged in the wireless communication system.

The founders of MIMO theory proved mathematically on the basis of Shannon formula that MIMO techniques, i.e. multi-transmitting antennas and multi-receiving antennas to be used at the same time, performed perfectly in the improving of $\eta_{f}$ compared with the other techniques, and also derived the MIMO CC formula similar to the Shannon formula. Pointed out by [2, 3, 4], MIMO technique is more suitable to be used in flat fading channel or frequency selective fading channel, especially in multi-scattering channels. Actually, MIMO technique tries to improve CC via the second definition for the $\eta_{p}$ increasing, which is to gain better SNR by the diversity receiving of those multi-path propagation channels. It is a pity that the above important physical concepts have not been given in [2], and so the physical model of MIMO. Also the mutual interferences among the signals transmitted by multi-antenna in the receiver are not concerned in [2]. MIMO techniques could be split into two groups basically: STC (Space Time Coding) [4] and SDM (Space Division Multiplexing) [2, 3, 4]. Channel coding over different transmitting branches is used to increase CC by STC technique, whereas SDM achieves its CC gain by transmitting same information on independent Rayleigh fading channel (IRFC) between multi-pair of transmitting and receiving antennas. It is sure that the CC gain got by SDM is introduced by multi-antenna, and it is fit with the original definition of MIMO [2].

In [2], MIMO theory is analyzed and proved in baseband and the physical model, so that The SDM is used in MIMO system to increase its CC in [2] without using the concept of phase controlled antenna (PCA). Furthermore, it is thought in [2] that the condition for IRFC between a pair of transmitting and receiving antennas is always satisfied when spacing between neighboring antennas is the half of wavelength $(\lambda)$ or $10 \lambda$. The above standpoints are unsuitable.

In [4], an explicit formula is given for the relation between CC and the number of antennas, i.e. MIMO CC formula, so as the physical model of MIMO. Based on the CC formula in [4] and the effect of beam forming by multiantennas mentioned above, it can be judged: Although the correctness of that the CC may be increased by MIMO theory is proved mathematically, it can not be realized physically, since the physical characteristics of antenna array has not been considered in the certifying. The above problems have been discussed in [5, 6].

$[3,7]$ have tried to prove the feasibility of MIMO system by experimental method. It could be found that the testing method is unreasonable and the conclusion was not convictable. 


\section{BeAm-FORMing PATterns OF MUlti-ANTENNA}

Some transmitting radio wave patterns for some antenna arrays to be used in [2, 3, 4] would be given in this section. The simulation method for these patterns was mentioned in [8]. Since the phase of signal fed to each antenna or array elements (AE) has not been controlled in MIMO system, so assuming that all of the phases for different $\mathrm{AE}$ are equal, the assumption is reasonable because even if they are different, the difference between them would keep unchanging, and similar patterns of radio wave beams could be obtained. While in PCA system, the phase of the signal fed to each AE is controlled, making the receiving signals from those AEs in Tx with the same phase, then they could be added in-phase, and SNR gain is increased sharply. The interposal effect or multi-path fading (MPF) between radio waves transmitted by different AE is inevitable in MIMO system, which would produce the constructive or destructive fading, and make the radio power non-uniform greatly in different directions. When the destructive fading caused, the SNR in some directions would become small enough, and CC value of the user in that direction would tend to 0. Moreover, it is useless to use any form of receivers when the MPF caused by multiantenna in transmitter leads to non-signal at the receivers.

Beam patterns of two kinds of multi-antenna used in [2] are given in Fig. 1. It is shown in Fig. 1(a) that SNR is nearly 0 in the direction of the connecting line between these two AEs, i.e. in the direction of $0^{\circ}$ and $180^{\circ}$, making $R_{b} \approx 0$. The communication would be failed in more than half area, if this kind of MIMO system to be used. The rectangular 4$\mathrm{AE}$ antenna array is used in Fig. 1(b), and it is shown that users in the direction of $0^{\circ}$ and $90^{\circ}$ can't communicate normally, too.

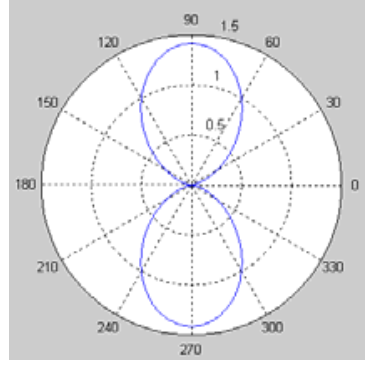

(a). 2AEs with $\lambda / 2$ spacing

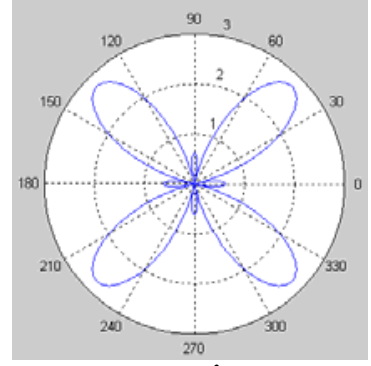

(b). 4AEs with $\lambda / 2$ spacing
Figure 1. Beam patterns of 2 and 4 antennas

In Fig. 2, there is the beam pattern of 2-AE spaced by $10 \lambda$, and there are also many directions with the intensity of signal equal to zero.

The beam pattern of 3 antennas with $2 \lambda$ spacing in [3] is shown in Fig. 3, and there are also many directions with transmission power to be equal to zero. The condition of spacing between neighboring antennas has not been given in [4], so the simulation curves given by it are with no meaning.

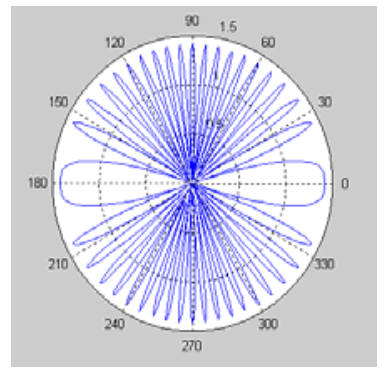

Figure 2. 2-AE with $10 \lambda$ spacing

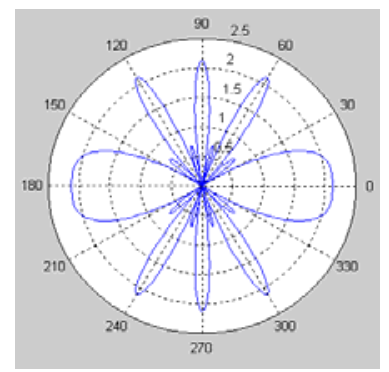

Figure 3. 3-AE line array with $2 \lambda$

It is shown by the beam patterns for many kinds of antenna array, which were taken as examples by those references listed in the paper, that the normal requirement for the receiving SNR in large area could not be satisfied in the MIMO system, existing serious technique defect, so the correctness of MIMO theory should be researched and proved further.

\section{THE ANALYZING OF THE MIMO PHYSICAL MODEL AND ITS TECHNIQUE DEFECTS}

In this part, some reasonless parts in MIMO theory and some reference papers would be analyzed continuously.

The explicit MIMO CC formula given in [4] is as follows, $C=N \log _{2}(1+\rho) \quad b p s / H z$.

where $N=N_{t}=N_{r}$ represents number of antennas in Tx and $\mathrm{Rx}$, the physical meaning of this formula should be that the receiving SNR, which is obtained by the diversity transmitting and receiving under the condition of that the transmitting power of all the AEs is equal to that of single antenna, should be proportional to the increasing of the number of antennas. Then $\eta_{p}$ gain by the 2 nd definition should be obtained which may be used to exchange for the gain of $\eta_{f}$. There is only the SNR factor, also which is the only physical quantity in MIMO formula similar to Shannon formula, could be used to improve CC. But actually, $\rho$ could be increased only when PCA is used to produce directional narrow beams, making the covering area to be reduced and the radio power to be concentrated. The physical model of MIMO system was given in [4], too, which is shown in Fig. 4. Those input signals in the left of Fig. 4 should be the same, i.e. $S_{1}=S_{2}=\ldots=S_{N_{t}}$. 
According to the basic theory of MIMO, transfer matrix $\mathrm{H}$ in Fig. 4 expresses those independent random channels between multi-pair of Tx and Rx antennas. But the effect of different antenna types hasn't been considered in the model, which is not suitable for the parabola antenna, being only fit for the omni-directional antenna. The basic problem is that the model did not fit the surroundings with multi-path propagation or multiple scattering in room, which are those environments designated by the basic theory of MIMO [2, 4]. It is because that those channels formed by scattering and multi-path propagation were not depicted in the physical model or included in the matrix $\mathrm{H}$. Considering the effect of multi-path propagation, the total number of channel $\mathrm{M}$, being given in the later, should be increased by several times. Supposing that those Tx antennas in the left side of Fig. 4 are substituted by the scattering sources or reflecting bodies, the similar physical model of MIMO in Fig. 4 could still be obtained even if without multi-antenna for Tx, and as a result, it is need not to assign the total transmitting power to $N_{t}$ antennas for $\mathrm{Tx}$, and the number of channels need to be processed is reduced, so that the receiver circuit could be simplified sharply and the probability of MPF would be fall, too. It will repudiate the necessity to use multi-antenna for Tx and the correctness of the physical model for MIMO, making the basis of MIMO theory unstable.

According to the physical model in Fig. 4, it is defined in $[2,4]$ that Multi Antennas Processing Unit (MAPU) must be used to finish the channel estimation and receiving process of those $M=N_{t} \times N_{r}$ IRFCs in $\vec{H}$, and then adjust those time delays among $M$-path receiving signals, combining them by the criteria of maximum SNR ratio (MRC) to make use of the energies of multi-path signals. Although the above processing can be expressed by a simple matrix, it is a very hard problem to be solved by the modern radio communication technique, since it is restricted by the physical feature of the antenna array.

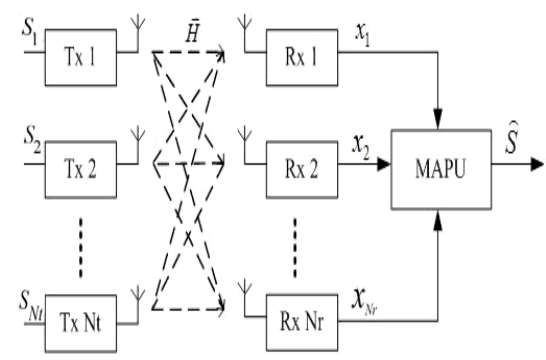

Figure 4. Physical model of MIMO system

Apart from the above problem, it's shown in Fig. 4 that there are unexpected $N_{t}-1$ path signals from $N_{t}$ Tx antennas included in the receiving signal $x_{1}$ of antenna $R x_{1}$ must be reduced from $x_{1}$ to form IRFC between $T x_{1}$ and $R x_{1}$. It has not been considered for how to make use of the energies of those $N_{t}-1$ path unexpected signals, so as the noise restraining problem for those unexpected signals. It is mentioned in [2] that joint receiver (JD) used in CDMA could be used to finish that process, though JD could be realized for the surrounding with multi-path propagation in CDMA system in theory, and there are still many problems to be resolved. How to solve these problems have not been mentioned by the other MIMO references such as [3, 4], so correctness of those conclusions given by them should be researched again.

It should be emphasized that the surrounding condition for the JD realization is the coarse multi-path propagation surrounding, its physical meaning is that the time delay between two-path signals must be longer than a CDMA chip. The other condition is that JD should be used in spectrum spreading system. Delay between two multi-path signals introduced by two Tx antennas is very small since the spacing between them is also very small, belonging to thin multi-path propagation surrounding, so as for the indoor scattering propagation. In these cases, the characteristic of multi-path channels could not be detected by training sequence or pilot with longer symbol duration, so the required function of JD could not be realized in these situations.

The channel bandwidth of general OFDM system is about $20 \mathrm{KHz}$, the time of the symbol in the prefix, which may be used for the detecting of the characteristic of multipath propagation, is too long for the detecting, so it is too hard to realize JD in OFDM system. However, all of the papers used to prove the correctness of MIMO system are based on OFDM system. By the discussion above, It is sure that the basic condition of M IRFCs in MIMO theory is too hard to be satisfied.

It was often mentioned in $[2,4]$ that SDM and SDMA can be realized by MIMO technique, however it is a unsuitable standpoint. It is impossible to realize SDM in physical realm for MIMO system, since the directional transmitting, which must be realized by PCA, has not been used in it. According to the physical model in Fig. 4, it only tries to set up IRFC by base-band processing, which also have been proved being impossible in previous part.

The key difference between SDM and SDMA is defined in [4], i.e. that SDMA allows each user to transmitting signal by single antenna one's self simultaneously, while SDM requires user to do this with multiple antennas. Comparing with the concepts of TDMA and TDM, or CDMA and CDM, the definition about SDM and SDMA in [4] is unsuitable. They are important concepts in MIMO system, but more confusion has been made on them by MIMO system.

\section{RESEARCH ON THE EXPERIMENTS OF MIMO}

There are not so many papers proving the usability of MIMO theory, and almost all of the proving processes are unreasonable. In [2], the check of CC improving was calculated through the derived MIMO formula only. Considering the physical characteristics of multi antennas, it is not suitable to express the CC of a real system by the computing value of MIMO formula. 
In [3], using the self-developed OFDM equipment to prove the feasibility of MIMO theory, however, it violated the basic application principles of Shannon formula, when $N_{t}=N_{r}=3$ and $R_{b}=108 \mathrm{Mbps}$, the selected modulation scheme was 16QAM; while in case of $N_{t}=N_{r}=1$ and $R_{b}=54 \mathrm{Mbps}$, that was 64QAM, this will result in different symbol rate for those two modulation schemes, so different channel bandwidths are needed for them. It is not allowed for the proving of CC gain by MIMO theory, so the performance test method is wrong, and its conclusions are meaningless.

Spacing between neighboring antennas was not given in the performance simulation of OFDM-MIMO in [4], selecting $N_{t}=N_{r}=2$ and the modulation scheme selected always being QPSK, which also violated the basic application principles of Shannon formula, and the CC gain by MIMO theory has not been checked by its simulation results, so its conclusions are meaningless, too.

Testing conditions of OFDM-MIMO system given in [7] are that 2 sets of directional antenna for Tx with antenna gain $G_{A T}$ of $16 \mathrm{dBi}$ and the beam width being $60^{\circ}$ for $3 \mathrm{~dB}$ respectively, and 3 sets of directional antenna with $1.5 \lambda$ spacing for Rx with antenna gain $G_{A R}$ of $12 \mathrm{dBi}$ and the $3 \mathrm{~dB}$ bandwidth being $60^{\circ}$ respectively. Apparently, there is an error in the given testing condition, where the $3 \mathrm{~dB}$ bandwidth should be corrected as $3 \mathrm{~dB}$ beam width. It is very strange for the directional antenna to be used in MIMO system, really it is not allowed. But the position relation among multi-antenna was not mentioned in [7].

Experiment for proving the feasibility of MIMO theory in [7] made the same elementary fault as that in [3], it was mentioned that CC gain in 2 times could be obtained when using SDM mode, e.g. 2 sets of antennas being used and using the same modulation scheme as that for single antenna system. Further more, it was discussed in [5] that delay diversity was used in 2 antennas, i.e. 2 transmitting signals had relative time delay, and frequency-selective fading would be introduced but space-frequency diversity gain should be obtained. However, this relative delay diversity between the 2 signals transmitted by 2 antennas should not introduce frequency-selective fading, which should be determined by the phase difference between 2 transmitting signals. This delay diversity can not obtain the gain of frequency diversity in space-frequency diversity, because frequency diversity transmitting must use 2 different carrier frequencies. The gain of space diversity also can not be obtained since the premise of obtaining space diversity gain is that 2 antennas must send the same signal. The delay diversity in [7] would produce 2-path signals with relative delay in the received signal, introducing serious interinterference.

\section{SUMMARY}

MIMO theory tries to establish IRFC by JD realized in base-band, and then realize SDM and diversity receiving of multi-channels to improve channel capacity. IRFC based on base-band do not fit the basic physical concept of SDM. The only method to realize SDM is to use PCA to generate directional narrow beams, so SDM defined by MIMO can not be realized.

The effect of scattering and multi-path propagation has not been considered in MIMO physical model. That physical model for MIMO was unreasonable since the number of channels introduced by radio propagation channels would be up to several times of those defined by MIMO.

Multi-antenna are introduced in MIMO system to create multi-channel, it will waste expensive radio power to create thin multi-path interference in receiver. MIMO tries to use JD to realize diversity receiving of multi-path signals, but the time delay between two thin multi-path signals is too small for the realization of JD. While in multi-path propagation environment, the delay between two rough multi-path signals is long enough to do the RAKE receiver or JD, which could be used to collect the energies of different path signals, making $\eta_{p}$ increased.

In conclusion, it is obvious that MIMO theory doesn't match its application environment physically. The infeasibility of MIMO is proved in the aspects of physical characteristic of antenna, radio beam patterns of several antenna array, un-reasonability of MIMO physical model, infeasibilities of IRFC and SDM in MIMO theory, etc.

\section{ACKNOWLEDGMENT}

This work was supported by the Research Fund for the Doctoral Program of Higher Education of China under Grant 20113223110001.

\section{REFERENCES}

[1] Haiyang Fu, Longxiang Yang, Wenglong Li, Modern Telecommunication Transmission, Posts and Telecommunication Press, Beijing, 2001. 2.

[2] G. J. Foschini and M. J. Gans, "On limits of Wireless Communications in a Fading Environment When Using Multiple Antennas," Wireless Pers. Commun., vol. 6, No. 3(Mar. 1998), pp. 311-335.

[3] Allert van Zelst and Tim C. W. Schenk, "Implementation of a MIMO OFDM-Based Wireless LAN System," IEEE Transaction on Signal Processing, vol. 52, No. 2(Feb. 2004), pp. 483-494.

[4] A. van Zelst, R. van Nee, and G. A. Awater, "Space Division Multiplexing (SDM) for OFDM Systems," in Proc. IEEE Veh. Technol. Conf.(May. 2000), pp. 1070-1074.

[5] JIA Xiang-dong, Chen Ji-jiang, Fang Hui, FU Hai-yang, "Analysis of MIMO Systems in Practical Engineering Implementations and 3Dimensional Channel Capacity Formula for SHPCA Multipleantenna systems," Signal Processing, Vol. 27(8), Aug. 2011, pp. 1160-1165.

[6] FU Hai-yang, Chen Ji-jiang, CAO Shi-ke, JIA Xiang-dong, "Study on the MIMO System and Wireless Channel Capacity," ACTA ELECTRONNICA SINICA, Vol. 39, No. 10, Oct. 2011, pp. 22212229.

[7] H. Sampath, S. Talwar, J. Tellado, V. Erceg and A. Paulraj, "A Fourth-Generation MIMO-OFDM: Broadband Wireless System: Design, Performance, and Field Trial Results," IEEE Commun. Mag., Vol. 40, No. 9(Sept. 2002), pp. 143-149.

[8] Jia Xiangdong, Li Fan, Zhen Jianguang, "Scheme of Beam-forming for Smart Antenna by Using Base-Band Amplitude Weighing and Performance Analysis in CDMA," Chinese Journal of Radio Science, Vol. 25, No. 3(2010. 6), pp. 505-511. 